González, A. D. y Varela, N. Violencia unificada y justicia fragmentada: Un análisis crítico de las trayectorias de las causas de violencia de género. Derecho y Ciencias Sociales. Mayo-Octubre 2019. № 21. Pgs 186-213. ISNN 1852-2971. Instituto de Cultura Jurídica y Maestría en Sociología Jurídica. FCJ y S. UNLP

\title{
Violencia unificada y justicia fragmentada: Un análisis crítico de las trayectorias de las causas de violencia de género
}

\author{
Unified violence and fragmented justice: A critical analysis of the trajectories of the causes of \\ gender violence
}

\author{
Agostina Daniela González y Natalia Varela
}

\section{Resumen}

En el presente trabajo nos proponemos analizar uno de los aspectos de la falta de efectividad de la respuesta estatal ante los casos de violencia de género brindada en sede jurisdiccional. En este sentido, nos enfocaremos en la problemática de la fragmentación de las causas entre el fuero civil y el fuero penal en relación a determinadas situaciones de violencia que son abarcadas tanto por la legislación penal como civil o de familia simultáneamente, así como también en la falta de especialización en temáticas relacionadas.

Para ello, nuestro enfoque teórico se apoyará fundamentalmente en las teorías críticas feministas del derecho que parten de considerar la situación de desigualdad estructural en la cual se encuentra la mujer por ser un grupo históricamente segregado y al derecho como una herramienta de perpetración de dicha desigualdad en tanto no se lo aplique con una perspectiva de género. La estrategia metodológica utilizada consiste, principalmente, en la confección de un análisis normativo enriquecido por el análisis de criterios jurisprudenciales para obtener una mejor comprensión del alcance real de las normas en materia de violencia contra las mujeres.

A continuación, a través del análisis documental de informes de instituciones que procesan datos relacionados a la temática y de organizaciones que la monitorean, explicaremos la trayectoria de las causas de violencia género que no tienen competencia exclusiva en ningún fuero específico y detectaremos algunos de los problemas que deben enfrentar las víctimas de violencia de género cuando deciden judicializar estos hechos. Por último esbozaremos algunas reflexiones para crear alternativas que mejoren la administración de justicia en la temática.

Palabras clave: violencia de género, judicialización, tutela judicial efectiva, perspectiva de género.

\begin{abstract}
Our propose is to analyze one of the aspects comprised in the lack of effectiveness as regards the degree of the Government's participation in gender violence cases. Therefore, we focus on criminal and civil proceedings in connection with certain circumstances where violence is present, as well as the lack of expertise in related subjects. For this purpose, our theoretical approach is fundamentally based on critical feminist legal studies founded on considering the situation of structural inequality in which women find themselves as a historically segregated group and the law as a tool for perpetration of such inequality as long as it is not applied with a gender perspective. The methodological strategy used mainly consists in regulations and jurisprudence analysis to obtain a better understanding of the real application of the regulations on violence against women.

Then, through the documentary analysis of reports from institutions that process information related to the topic and on the organizations that monitor it, we will explain the trajectory of the causes of gender violence that do not have exclusive competence in any specific jurisdiction and we will detect some of the problems that the victims of gender violence must face when they decide to judicialize this issue. Finally, we will outline some considerations to create alternatives that improve the administration of justice.
\end{abstract}

Key words: gender violence, judicial proceedings, effective legal protection, gender perspective.

•Integrantes del equipo DCT1801 2018-2020 (UBA, Facultad de Derecho). Becarias EVC-CIN 2017. Ex becarias PII
2017-2018. agostinadgonzalez@hotmail.com; varelanatalia1@gmail.com

Recibido:11/9/2018. Publicable con correcciones : 14/8/2019 
González, A. D. y Varela, N. Violencia unificada y justicia fragmentada: Un análisis crítico de las trayectorias de las causas de violencia de género. Derecho y Ciencias Sociales. Mayo-Octubre 2019. № 21. Pgs 186-213. ISNN 1852-2971. Instituto de Cultura Jurídica y Maestría en Sociología Jurídica. FCJ y S. UNLP

\title{
Violencia unificada y justicia fragmentada: Un análisis crítico de las trayectorias de las causas de violencia de género ${ }^{1}$
}

\author{
Agostina Daniela González y Natalia Varela
}

\section{Introducción}

Históricamente, las mujeres han sido un grupo segregado y excluido de ciertos ámbitos, como el mercado laboral, la actividad política o la educación superior, siendo asignadas con roles estereotipados en razón de su género, limitados a las tareas de cuidado o las actividades domésticas, encontrándose así en una situación de desigualdad estructural. La limitación a la autonomía y libertad de las mujeres tiene, como una de sus manifestaciones, el ejercicio de violencia contra ellas, que se presenta en diversas dimensiones y contextos, pero tiene como origen la desigualdad estructural en la que se encuentran inmersas (Bodelón, 1995) ${ }^{2}$. En este sentido, la Declaración de Beijing considera que la violencia contra la mujer es "todo acto de violencia basado en el género que tiene como resultado posible o real un daño físico, sexual o psicológico, incluidas las amenazas, la coerción o la privación arbitraria de la libertad, ya sea que ocurra en la vida pública o en la privada”. Asimismo, la Declaración sobre la Eliminación de la Violencia contra la Mujer, ha reconocido que

"la violencia contra la mujer constituye una manifestación de relaciones de poder históricamente desiguales entre el hombre y la mujer, que han conducido a la dominación de la mujer y a la discriminación en su contra por parte del hombre e impedido el adelanto pleno de la mujer, y que la violencia contra la mujer es uno de los mecanismos sociales fundamentales por los que se fuerza a la mujer a una situación de subordinación respecto del hombre".

Esta situación puede verse agravada por la presencia de otros factores de discriminación, como la clase o la etnia. Asimismo, las mujeres que pertenecen a grupos sociales insertos en una vulnerabilidad mayor (pobreza, desocupación, niñez, entre otros), se encuentran expuestas a mayor violencia ${ }^{3}$.

\footnotetext{
${ }^{1}$ Una primera versión de este trabajo fue presentada en las V Jornadas de Jóvenes Investigadoras/es en Derecho y Ciencias Sociales 2017 del Instituto de Investigaciones Jurídicas y Sociales Ambrosio L. Gioja. Agradecemos los comentarios y sugerencias de Martín M. Aldao, Celeste Novelli, Victoria Meza, Victoria Ricciardi y Nadia Ferrari.

${ }^{2}$ Para un análisis más profundo de la dimensión histórica del carácter estructural de la desigualdad y de la violencia de género, ver Federici (2005).

${ }^{3}$ Al respecto, véase Sosa-Sánchez (2017) y Amnistía Internacional (2007).
} 
González, A. D. y Varela, N. Violencia unificada y justicia fragmentada: Un análisis crítico de las trayectorias de las causas de violencia de género. Derecho y Ciencias Sociales. Mayo-Octubre 2019. № 21. Pgs 186-213. ISNN 1852-2971. Instituto de Cultura Jurídica y Maestría en Sociología Jurídica. FCJ y S. UNLP

Ahora bien, durante siglos, los ámbitos de conocimiento considerados "legítimos" estaban compuestos casi exclusivamente por hombres, produciéndose una marginación de las mujeres. Este fenómeno se replicó también en la construcción del derecho: éste es un producto de la cultura masculina en cuanto a sus contenidos (las normas jurídicas), pero a su vez estos también se han construido en base a valores predominantemente masculinos, son aplicadas e interpretadas mayoritariamente por hombres y por lo general, su punto de vista excluye el de las mujeres (Facchi, 2005, p. 35). Considerando la relevancia de las respuestas judiciales ante un caso de violencia de género, no es un dato menor que desde los orígenes del derecho, el androcentrismo jurídico haya tenido como objetivo la consolidación de los privilegios y la dominación masculina por sobre cualquier otro grupo (Costa, 2017, p. 238). Por ello, es que para realizar el análisis propuesto, debemos partir de que el derecho ha sido una institución que históricamente ha contribuido a mantener y reproducir el estatus de inferioridad de las mujeres (Facio y Fries, 2005, p. 260).

De un tiempo a esta parte, el debate sobre cuestiones vinculadas a la igualdad de género ha tomado mayor visibilidad. Así, luego de muchos años de lucha por parte de los movimientos feministas, se logró que sea debatida por primera vez en el Congreso de la Nación la ley de interrupción voluntaria del embarazo en 2018. Tras un debate histórico, se logró la media sanción en la Cámara de Diputados, aunque el proyecto no prosperó en la Cámara de Senadores. Sin embargo, se trató de un año bisagra en el que se puso en discusión una de las principales demandas feministas, contando con las exposiciones de más de 300 expertas/os en el tema en el Congreso, pero también con discusiones y exposiciones mediáticas sobre el tema ${ }^{4}$.

En lo que hace a la violencia contra la mujer, es posible afirmar que ha tomado un lugar relevante en los medios de comunicación ${ }^{5}$ pero también en las calles con las marchas masivas que se han realizado en los últimos años en reclamo a una respuesta ante este flagelo. De esta manera, los femicidios que tuvieron como víctimas a Lucía Perez y Micaela García, sólo por nombrar dos casos de gran relevancia, pusieron en el centro de la escena el debate no sólosobre la violencia contra la mujer, sino el rol del Poder Judicial en su intervención frente a este tipo de violencia. Como resultado de ello, no solo se gestaron masivas marchas signadas por reclamos de

\footnotetext{
${ }^{4}$ Para una muestra del impacto que tuvo este debate en la sociedad, véase Luciana Sánchez (2018). "Desde que Señorita Bimbo habló en Intrusos sobre el Misoprostol, la palabra explotó en Twitter, en el buscador de Google y en Wikipedia".

${ }^{5}$ En este sentido, con el auge de los movimientos feministas los medios de comunicación comenzaron un proceso de "deconstrucción" machista, por ejemplo reemplazando el uso del término "crimen pasional" para referirse a un femicidio. Sobre este punto, véanse algunas reflexiones en Agustina Paz Frontera, 2019).
} 
González, A. D. y Varela, N. Violencia unificada y justicia fragmentada: Un análisis crítico de las trayectorias de las causas de violencia de género. Derecho y Ciencias Sociales. Mayo-Octubre 2019. № 21. Pgs 186-213. ISNN 1852-2971. Instituto de Cultura Jurídica y Maestría en Sociología Jurídica. FCJ y S. UNLP

“justicia", sino que también esto tuvo un impacto directo en la generación de políticas públicas del Estado acerca de la violencia de género ${ }^{7}$.

Por su parte, la denuncia en el exterior y exposición en los medios masivos de comunicación del hecho de violación que tuvo como víctima a la actriz Thelma Fardin abrió el debate sobre los distintos obstáculos que deben sortear las víctimas de delitos sexuales para poder realizar la denuncia, y la revictimización a que son sometidas una vez que lo logran. Es importante destacar que luego de que la actriz hiciera público el hecho del que resultó víctima hubo un aumento exponencial en las llamadas a la línea telefónica $144^{8}$ de denuncias por delitos sexuales. ${ }^{9}$

Los ejemplos a los que hicimos mención en estas líneas y la reacción social generada luego de que tomen estado público, son algunas de las causas que nos hacen cuestionar la idoneidad de la labor judicial frente a la violencia de género. A continuación, esbozaremos un recorrido por el marco normativo y jurisprudencial en la materia.

\section{Marco normativo y jurisprudencial}

Tanto a nivel internacional como regional, se han sancionado diversas normas que abordan la problemática de la violencia de género. En cuanto al sistema universal, el instrumento más importante es la Convención sobre la Eliminación de todas las Formas de Discriminación contra la Mujer (en adelante CEDAW), sancionada en 1979, la cual afirma el principio de igualdad y se concentra en tres aspectos de la situación de la mujer: los derechos civiles, el derecho de procreación y las consecuencias de los factores culturales en las relaciones entre los sexos. Asimismo, el Comité CEDAW emitió en 1992 la Recomendación General Número 19, donde define con mayor especificidad el concepto de violencia de género que incluía la CEDAW, siendo un importante antecedente para la sanción de la Convención Interamericana para Prevenir, Sancionar y Erradicar la Violencia contra la Mujer (en adelante Convención de Belém Do Pará). Así, en el sistema interamericano, se encuentra la Convención de Belém Do Pará sancionada en

\footnotetext{
${ }^{6}$ Véase sobre la marcha masiva ante la absolución de los acusados por el caso de Lucía Pérez, Fernanda Jara (5 de diciembre de 2018).

${ }^{7}$ Por ejemplo, la ley 27.499 sobre capacitación en género para todas las personas que integran los tres poderes del Estado fue denominada "Micaela" por el caso del brutal femicidio mencionado en este acápite. Sobre este punto profundizaremos más adelante.

${ }^{8}$ La línea telefónica nacional y gratuita 144 fue creada en el año 2013 con la finalidad de brindar contención, información y asesoramiento en violencia de género y depende del Consejo Nacional de Mujeres.

${ }^{9}$ Véase sobre el aumento de denuncias tras la denuncia de Thelma Fardin. Martín Slipczuk (19 de diciembre de 2018).; Infobae (12 de diciembre de 2018); entre otras.
} 
González, A. D. y Varela, N. Violencia unificada y justicia fragmentada: Un análisis crítico de las trayectorias de las causas de violencia de género. Derecho y Ciencias Sociales. Mayo-Octubre 2019. № 21. Pgs 186-213. ISNN 1852-2971. Instituto de Cultura Jurídica y Maestría en Sociología Jurídica. FCJ y S. UNLP

1994, que establece, principalmente, el estándar de "debida diligencia" a cargo del Estado respecto de la prevención y sanción de la violencia contra las mujeres. Por otro lado, reconoce que la violencia contra la mujer parte de relaciones de poder históricamente desiguales entre mujeres y hombres.

Argentina ha adoptado diversas normas tanto nacionales como internacionales que buscan proteger a las mujeres ante la situación de desigualdad en la que se encuentran: a nivel constitucional, se encuentra la CEDAW, incorporada en 1994 al artículo 75 inciso 22 de la Constitución Nacional, la Convención de Belém Do Pará, ratificada por Argentina en 1996 mediante la sanción de la ley 26.632; la ley 24.417 de protección contra la violencia familiar, que protege a "(t)oda persona que sufriese lesiones o maltrato físico o psíquico por parte de alguno de los integrantes del grupo familiar" (cfr. art. 1), sancionada en 1996; la ley 26.485 de protección integral para prevenir, sancionar y erradicar la violencia contra las mujeres en los ámbitos en que desarrollen sus relaciones interpersonales, sancionada en 2009; la ley 26.791 sancionada en 2012, a través de la cual se produjo la incorporación de la figura del femicidio como agravante de la figura del homicidio simple, toda vez que fuera cometido en razón de la condición de mujer de la víctima; entre otras ${ }^{10}$.

Por otro lado, hay un diverso desarrollo jurisprudencial en la materia. En el ámbito internacional, se destacan varios precedentes. En cuanto a la Comisión Interamericana de Derechos Humanos (en adelante $\mathrm{CIDH}$ ), hay dos casos muy importantes sobre violencia cometida por los particulares: el caso María Da Penha c. Brasil (2001) y el caso Jessica Lenahan c. EEUU (2011). En el primero de ellos, la CIDH concluye que el Estado brasileño es responsable de haber vulnerado las garantías judiciales garantizadas por los artículos 8 y 25 de la Convención Americana de Derechos Humanos (en adelante $\mathrm{CADH}$ ) así como también la obligación general de respetar y garantizar los derechos, prevista en el artículo 1 de dicho instrumento, por la dilación injustificada y tramitación negligente del caso en cuestión sobre violencia doméstica. Asimismo, indica que las medidas llevadas a cabo por Brasil para disminuir la violencia contra la mujer son ineficaces, ya que no han logrado reducir de manera significativa el patrón de tolerancia estatal, en particular por la inefectividad de la acción judicial respecto de los casos de violencia contra la mujer. En lo que interesa al presente trabajo es necesario destacar que dos de las recomendaciones efectuadas por la CIDH a la República Federativa de Brasil en el mentado caso, a fin de "(c)ontinuar y profundizar

\footnotetext{
${ }^{10}$ Por otro lado, a nivel provincial se destacan, mayoritariamente, las leyes específicas referidas a la violencia familiar, la creación de leyes procedimentales específicas para tramitar los casos de violencia y, en algunos casos, la creación de fueros especializados (por ejemplo, la ley 7.184 de Santiago del Estero).
} 
González, A. D. y Varela, N. Violencia unificada y justicia fragmentada: Un análisis crítico de las trayectorias de las causas de violencia de género. Derecho y Ciencias Sociales. Mayo-Octubre 2019. № 21. Pgs 186-213. ISNN 1852-2971. Instituto de Cultura Jurídica y Maestría en Sociología Jurídica. FCJ y S. UNLP

el proceso de reformas que eviten la tolerancia estatal y el tratamiento discriminatorio respecto a la violencia doméstica contra las mujeres", establecen que el Estado brasileño debe llevar adelante “(m)edidas de capacitación y sensibilización de los funcionarios judiciales y policiales especializados para que comprendan la importancia de no tolerar la violencia doméstica” y “simplificar los procedimientos judiciales penales a fin de que puedan reducirse los tiempos procesales, sin afectar los derechos y garantías de debido proceso"11.

En el caso Jessica Lenahan c. EEUU, la CIDH mantiene y refuerza el criterio que había adoptado hacía diez años en el precedente mencionado anteriormente. Este caso se trataba puntualmente de una ciudadana estadounidense, Jessica Lenahan, quien denunció que la policía no había respondido a sus reiteradas y urgentes llamadas en las que denunciaba que su ex marido se había llevado a sus hijas, violando así una restricción perimetral, lo que derivó en la muerte de las niñas. El máximo tribunal de dicho país entendió que la policía actuó correctamente ya que no era su función controlar dicha restricción. Luego de un análisis del caso, la CIDH concluyó que el Estado no actuó con la debida diligencia para proteger a Jessica Lenahan y sus hijas de la violencia doméstica, en menoscabo de su obligación de no discriminar y de garantizar la igual protección ante la ley, conforme al artículo II de la Declaración Americana de los Derechos y Deberes del Hombre (en adelante Declaración Americana). El Estado tampoco adoptó medidas razonables para prevenir la muerte de las menores, en violación de su derecho a la vida, consagrado en el artículo I de la Declaración Americana, conjuntamente con su derecho a una protección especial como niñas, consagrado en el artículo VII de la Declaración Americana. Por último, la Comisión concluyó que el Estado violó el derecho a la protección judicial de Jessica Lenahan y de sus familiares, establecido en el artículo XVIII de la Declaración Americana, en virtud de lo cual, entre otras cosas, recomendó al Estado estadounidense adoptar una legislación con medidas integrales a nivel federal y estatal, las que deben ir acompañadas de suficientes recursos destinados a fomentar su implementación; de una reglamentación adecuada para garantizar su implementación; de programas de capacitación para los funcionarios policiales y judiciales involucrados; y del diseño de protocolos y directivas modelo que los departamentos de policía de todo el país puedan usar como guía.

Por otro lado, se encuentran las producciones jurisprudenciales de la Corte Interamericana de Derechos Humanos (en adelante Corte IDH), entre las que se destacan el caso Penal Miguel Castro Castro vs. Perú (2006), Campo Algodonero vs. México (2009), Véliz Franco vs. Guatemala

\footnotetext{
${ }^{11}$ Este fue un caso paradigmático en Brasil, a partir del cual se sancionó un nuevo marco legal sobre la violencia doméstica y familiar contra la mujer. Para profundizar esta cuestión, véase Maciel y Brito Prata (2011).
} 
González, A. D. y Varela, N. Violencia unificada y justicia fragmentada: Un análisis crítico de las trayectorias de las causas de violencia de género. Derecho y Ciencias Sociales. Mayo-Octubre 2019. № 21. Pgs 186-213. ISNN 1852-2971. Instituto de Cultura Jurídica y Maestría en Sociología Jurídica. FCJ y S. UNLP

(2014) y Velásquez Paiz vs. Guatemala (2015). El primer caso se trataba del “Operativo Mudanza 1" que las fuerzas de seguridad realizaron dentro del Penal Castro Castro, con el supuesto objetivo de trasladar a 90 internas a otro centro penitenciario femenino. La Policía Nacional derribó parte de una pared externa del patio de un pabellón utilizando explosivos, mientras abrieron boquetes en los techos del penal, desde donde realizaron disparos con armas de fuego, utilizando, a su vez, armas de guerra, explosivos, bombas lacrimógenas, entre otros elementos. Esta operación generó muchas muertes y heridos/as, mientras que los/as sobrevivientes fueron sometidos/as a golpes, agresiones y falta de atención médica. En cuanto a lo que nos interesa en este artículo, la Corte entendió que el Estado peruano era responsable por la violación de actuar con la debida diligencia para prevenir, investigar y sancionar la violencia contra la mujer, en perjuicio de todas las internas. Además, abordó la violencia sexual perpetrada por agentes estatales, y reconoció que las consecuencias de la violencia sexual contra la mujer se ven agravadas en los casos de las mujeres detenidas. La importancia de este caso radica en que, por primera vez, la Corte IDH habló de las especificidades de género en los hechos denunciados y determinó que poseía competencia para interpretar y aplicar la Convención de Belém do Pará.

En el caso Campo Algodonero vs. México, los hechos que dan origen a la denuncia fueron la desaparición y posterior muerte de tres jóvenes de Ciudad Juárez, cuyas familias recurrieron a los organismos estatales, los que subestimaron la situación y les solicitaron esperar 72 hs. para iniciar la búsqueda. En este caso, la Corte IDH abordó por primera vez el feminicidio como "homicidio de mujeres por razones de género". Consideró que las autoridades nacionales se limitaron a llevar a cabo labores formales administrativas y no medidas que lleven a encontrar a las víctimas; y que estos homicidios se habían producido por razones de género, en un contexto de violencia contra la mujer específicamente crítico en Ciudad Juárez. Así, la Corte consideró que el Estado de México no había actuado con la debida diligencia para prevenir, investigar y sancionar la violencia contra la mujer, tal como lo requiere la Convención de Belém do Pará, y que violó el deber de no discriminación contenido en el art. 1.1 CADH, ya que las víctimas tenían derecho a no ser discriminadas ni atacadas por su género.

Por su parte, en el caso Véliz Franco vs. Guatemala, la Sra. Sandoval había denunciado la desaparición de su hija ante la Policía Civil, sin que ningún funcionario iniciara acciones de búsqueda. Al día siguiente, encontraron el cadáver de su hija con signos de violencia y vejaciones. La Corte IDH consideró que el deber de garantizar los derechos humanos tiene como una de sus manifestaciones el deber de prevención, lo que había sido violado por Guatemala. Además, estimó que hubo falta de diligencia en la investigación, la cual estuvo vinculada a la ausencia de 
González, A. D. y Varela, N. Violencia unificada y justicia fragmentada: Un análisis crítico de las trayectorias de las causas de violencia de género. Derecho y Ciencias Sociales. Mayo-Octubre 2019. № 21. Pgs 186-213. ISNN 1852-2971. Instituto de Cultura Jurídica y Maestría en Sociología Jurídica. FCJ y S. UNLP

protocolos para la investigación de casos de violencia contra la mujer en general. Es decir, consideró la falta de diligencia del Estado en un caso en que el hecho había sido cometido por un particular. Por otro lado, entendió que el deber de no discriminación se vio agravado por el hecho de que funcionarios a cargo de la investigación efectuaron declaraciones que denotan la existencia de prejuicios y estereotipos sobre el rol social de las mujeres. Asimismo, remarcó el lapso excesivo de duración (más de doce años) de la investigación.

Finalmente, mencionaremos el caso Velásquez Paiz vs. Guatemala, cuyos hechos se enmarcan, según la Corte IDH, en "un contexto de aumento de la violencia homicida contra las mujeres en Guatemala”. Claudina Velásquez Paiz, luego de asistir a una fiesta, perdió contacto con su familia, la cual fue alertada de que ella se encontraba en peligro, por lo que acudieron a la Policía Nacional Civil, quienes les informaron que no podían hacer nada más que patrullar y que debían esperar 24 hs. para realizar la denuncia de desaparición. Al día siguiente, se encontró su cuerpo sin vida. No constaba que se haya desplegado actividad alguna de investigación a partir de las denuncias sobre su presentación, y la investigación penal comenzó recién con el hallazgo de su cuerpo. Nuevamente, en este caso se señala la falta de diligencia de las autoridades de Guatemala para prevenir la muerte de la víctima, que había sido perpetrada por un particular.

En cuanto al ámbito nacional, se destacan el fallo Góngora (2013) de la Corte Suprema de Justicia de la Nación (en adelante CSJN), por el cual entiende que es improcedente, a la luz de las obligaciones internacionales asumidas por nuestro país, la adopción de alternativas (como por ejemplo la suspensión del juicio a prueba) distintas al dictado de una sentencia, ya sea de condena o absolución, en los casos de violencia de género; así como también el fallo Fernández Corina c/ Weber Javier s/ tentativa de homicidio (2012) del Tribunal Oral en lo Criminal $\mathrm{n}^{\circ} 9$ (en adelante TOC 9) de la Capital Federal a través del cual se creó jurisprudencialmente lo que meses más tarde el Congreso de la Nación tipificaría como femicidio ${ }^{12}$. Dos de los jueces del TOC 9 en el caso mencionado aseguraban que

“(n)o cabe duda de que la muerte de una mujer a consecuencia de la violencia de género constituye una categoría sociológica claramente distinguible y que ha adquirido especificidad normativa a partir de la Convención de Belem do Pará. No hay razón, en consecuencia, para no darle nombre y, en tal sentido, cabe señalar que la conducta de Javier Claudio Weber constituye

\footnotetext{
${ }^{12}$ El fallo es del 8 de agosto de 2012 mientras que la ley que tipificó dicha figura se sancionó el 14 de noviembre del mismo año.
} 
González, A. D. y Varela, N. Violencia unificada y justicia fragmentada: Un análisis crítico de las trayectorias de las causas de violencia de género. Derecho y Ciencias Sociales. Mayo-Octubre 2019. № 21. Pgs 186-213. ISNN 1852-2971. Instituto de Cultura Jurídica y Maestría en Sociología Jurídica. FCJ y S. UNLP

un intento de femicidio, entendiendo por tal la muerte de una mujer $-\mathrm{o}$ de una persona con identidad femenina- ejecutada por un varón en razón del género”.

\section{La violencia machista en números: problemas e importancia}

El robusto marco normativo y jurisprudencial analizado en el punto anterior, así como la lucha que llevaron adelante las organizaciones sociales, hicieron posible la visibilización de distintas situaciones de violencia a las que históricamente se encuentran sometidas las mujeres y reflejan un punto de partida en la lucha contra la violencia machista. Sin embargo, las estadísticas continúan siendo alarmantes: según el $1^{\circ}$ Índice Nacional de Violencia Machista de 2016 "Contá la violencia machista", iniciativa impulsada por el movimiento "Ni Una Menos", el 97\% de las encuestadas alguna vez sufrió acoso en espacios públicos y/o privados, incluyendo haber sido tocada sin su consentimiento y recibir descalificaciones por ser mujer. Asimismo, al menos el $76 \%$ de las encuestadas ha sufrido amenazas e intimidaciones durante sus relaciones de pareja, mientras que el 67\% sufrió algún tipo de maltrato físico en la pareja (empujones, golpes con los puños u objetos, entre otros tipos) (Beck y Romeo, 2016).

La manifestación más grave de la violencia machista son los femicidios. Según el seguimiento realizado por la organización de la sociedad civil La Casa del Encuentro se registró un promedio de un femicidio cada 30 horas en nuestro país en el período que abarca del 1 de enero al 31 de diciembre de 2016 (Consejo Nacional de Mujeres, 2016).La cifra aumentó y entre el 1 de enero y el 31 de diciembre del año 2017: el observatorio de la violencia contra las mujeres del colectivo "Ni Una Menos" relevó 298 femicidios, lo que equivale a un femicidio cada 29 horas. Según el informe de dicho colectivo, el $90 \%$ de los femicidios fueron cometidos por hombres del círculo íntimo de la víctima, mientras que el $4 \%$ fueron cometidos por extraños, y del $6 \%$ restante no registran datos. Otra información de gran relevancia para el presente trabajo es que según el mencionado observatorio, el $18 \%$ de las víctimas de dichos femicidios habían realizado las denuncias previamente y en el $12 \%$ de los casos se habían dictado medidas de protecciónObservatorio Ni Una Menos (2018).

Finalmente, es necesario destacar que el primer registro oficial a nivel nacional sobre casos de violencia de género es el "Registro Único de Casos de Violencia contra las Mujeres" realizado por el Instituto Nacional de Estadísticas y Censos (en adelante INDEC), el cual fue publicado por primera vez en marzo del año 2018, y abarca el período comprendido entre el 1 de enero de 2013 hasta el 31 de diciembre de 2017. Es importante tener en cuenta que dicho registro no incorpora a su análisis los casos de femicidios, ya que éstos son captados en el "Registro Nacional de Femicidios de la Justicia Argentina" (CSJN, S/F), cuyo último informe corresponde al año 2017 
González, A. D. y Varela, N. Violencia unificada y justicia fragmentada: Un análisis crítico de las trayectorias de las causas de violencia de género. Derecho y Ciencias Sociales. Mayo-Octubre 2019. № 21. Pgs 186-213. ISNN 1852-2971. Instituto de Cultura Jurídica y Maestría en Sociología Jurídica. FCJ y S. UNLP

arroja un total de 251 femicidios directos, es decir tan sólo tres víctimas menos que el año anterior en el cual se registraron 254 femicidios.

La particularidad del registro del año 2017 radica en que por primera vez se han registrado los femicidios vinculados, es decir aquellos casos en los cuales el delito no se perpetúa contra la mujer víctima de violencia de género sino contra personas que mantienen un vínculo familiar o afectivo con ésta, con el objetivo de provocar dolor en la mujer. En este sentido, fueron contabilizados 22 casos durante el año 2017, lo que arroja una cifra final de 273 femicidios entre directos y vinculados. La existencia de registros oficiales es muy relevante no solo ya que para el planeamiento de cualquier política pública se requiere una previa elaboración de datos sobre la problemática, sino que luego también sirven para evaluar la eficacia de las medidas tomadas para prevenir, sancionar y erradicar la violencia de género. Asimismo, la necesidad de tener estadísticas había sido señalada por la Relatora Especial sobre la violencia contra la mujer en su último informe sobre Argentina, del año 2017, en el cual destacaba la importancia de "(r)ecolectar y publicar datos desglosados a nivel federal y provincial sobre todas las formas de violencia contra las mujeres..." (A/HRC/35/30/Add.3).

Ahora bien, la información con la que cuenta el mencionado registro surge a partir de los datos proporcionados por parte de los organismos públicos que registran casos de violencia contra las mujeres por razones de género. En este sentido, es importante resaltar que entiende por caso "todo hecho de violencia por cuestión de género, en el que medie una relación desigual de poder, informado o denunciado por una mujer o por terceros, personalmente o mediante un llamado telefónico, en el que la víctima solicite asesoramiento, asistencia, información o establezca una denuncia formal en la policía o inicie una causa judicial en los tribunales, entre otras acciones que puede llevar a cabo" (INDEC, 2018, p. 17). Esto implica que, si se tomara esta encuesta como punto de partida para diseñar políticas públicas, las medidas que se tomen para prevenir, erradicar y en la medida de lo posible reparar o aminorar los daños ocasionados por la violencia de género, serán diseñadas en base a los registros judiciales y/o policiales.

En este trabajo, no haremos un análisis pormenorizado del informe realizado por el INDEC ni analizaremos los problemas que sufren las mujeres víctimas de violencia de género en sede policial, pero sí es importante destacar el rol que se le otorga tanto al Poder Judicial como a la Policía, ya que son quienes deben registrar, primeramente, que un hecho denunciado se encuentra enmarcado en un contexto de violencia de género. Asimismo, que el rol del Poder Judicial adquiere aún más relevancia al ser una de los dos únicas fuentes de que se sirve el único registro oficial en la temática, ya que como vimos, los registros además de visibilizar una problemática que 
González, A. D. y Varela, N. Violencia unificada y justicia fragmentada: Un análisis crítico de las trayectorias de las causas de violencia de género. Derecho y Ciencias Sociales. Mayo-Octubre 2019. № 21. Pgs 186-213. ISNN 1852-2971. Instituto de Cultura Jurídica y Maestría en Sociología Jurídica. FCJ y S. UNLP

históricamente afectó a las mujeres del país, es fundamental para la implementación de políticas públicas. Esto pone de manifiesto la necesidad de repensar la manera en que se busca abordar la problemática de la violencia de género, para implementar nuevas políticas públicas que den respuesta a esta alarmante situación. Es decir, para avanzar en la prevención y erradicación de la violencia de género se necesita mucho más que la aprobación de marcos normativos o registros estadísticos, sino que además se requieren políticas públicas para asegurar su efectiva vigencia e implementación (ELA, 2012, p. 43) y ello se torna casi imposible si se desconoce la dimensión real de la problemática.

\section{Recorrido de las causas de violencia de género}

En este apartado, presentaremos un panorama general del procedimiento para tramitar causas de violencia. En primer lugar, debemos decir que existen múltiples vías en la Ciudad Autónoma de Buenos Aires (en adelante CABA) por las cuales las mujeres realizan consultas por episodios de violencia. Posteriormente, las consultas pueden convertirse en denuncias y en acciones judiciales en la justicia civil y/o penal. Las vías de consulta principales son las siguientes: Líneas telefónicas de asesoramiento ${ }^{13}$, Oficina de Violencia Doméstica (dependiente de la Corte Suprema de Justicia de la Nación, en adelante OVD), Comisión sobre Temáticas de Género de la Defensoría General de la Nación, Defensorías zonales, comisarías, Centros Integrales de la Mujer (dependientes del Gobierno de la CABA, en adelante CIM). Al mismo tiempo, en los últimos años se han creado diversas instituciones públicas cuyo objetivo es la promoción de políticas de equidad de género ${ }^{14}$. Esto se ha dado en el marco de una etapa de consolidación de las democracias y el afianzamiento en el reconocimiento de los derechos humanos, así como también resultaron centrales las reivindicaciones del movimiento de mujeres ${ }^{15}$, las feministas y el movimiento por la diversidad sexual (Bergallo y Moreno, 2017, p. 27), es decir que ha sido

\footnotetext{
13144 del Consejo Nacional de las Mujeres con competencia a nivel nacional; 0800-666-8537 - Línea MUJER y 137 del Programa Las Víctimas contra las Violencias del Ministerio de Justicia y Derechos Humanos de la Nación.

${ }^{14}$ Además de las mencionadas como vías de consulta, se encuentran el Observatorio de Género en la Justicia de la CABA, dependiente del Consejo de la Magistratura de la CABA, Oficina de la Mujer dependiente de la Corte Suprema de Justicia de la Nación, el Observatorio de Violencia de Género de la Defensoría del Pueblo de la Provincia de Buenos Aires, entre otras.

${ }^{15}$ Por ejemplo, en Argentina se ha destacado el colectivo Ni Una Menos, movimiento creado a raíz de la cantidad de femicidios que ocurren en el país por día, que ha convocado a marchas multitudinarias en los últimos años y cuya consigna se ha instalado en la agenda pública.
} 
González, A. D. y Varela, N. Violencia unificada y justicia fragmentada: Un análisis crítico de las trayectorias de las causas de violencia de género. Derecho y Ciencias Sociales. Mayo-Octubre 2019. № 21. Pgs 186-213. ISNN 1852-2971. Instituto de Cultura Jurídica y Maestría en Sociología Jurídica. FCJ y S. UNLP

fundamental la demanda tanto de la sociedad civil como de los organismos y movimientos sociales para la creación de estos espacios.

A pesar de esto, la situación de vulnerabilidad en la que se encuentran las mujeres víctimas de violencia de género hace que sea extremadamente difícil para ellas tomar la decisión de judicializar tales hechos, ya sea por vergüenza, por miedo, por falta de conocimiento de sus derechos y por otras circunstancias objetivas que obstaculizan tal decisión, como la dependencia económica, la marginalidad, el aislamiento, entre otras (ELA, 2009, p. 50). En este sentido, es importante la intervención de los actores sociales ${ }^{16}$, especialmente por sus tareas de asesoramiento, tratamiento, acompañamiento, difusión y visibilización de $\operatorname{casos}^{17}$. Asimismo, también suelen realizar tareas de investigación, colaboración en la creación de leyes y políticas públicas, realización de informes, elaboración de materiales informativos, entre otras.

Ahora bien, aquellas mujeres que toman la decisión de denunciar los hechos de los cuales son víctimas, deben además sortear obstáculos propios del sistema judicial, lo que muchas veces provoca que retiren sus denuncias luego de plantearlas. Al mismo tiempo, en otros casos, las mujeres que con anterioridad tienen conocimiento de los obstáculos que se generan con posterioridad a la denuncia, se abstienen de realizarla. El Comité CEDAW ha señalado en su Séptimo Informe periódico de la Argentina, que le preocupaban "las barreras institucionales, procedimentales y prácticas que dificultan el acceso de las mujeres a la justicia" (CEDAW/C/ARG/CO/7). Uno de dichos obstáculos se origina en aquellos casos en los cuales, los hechos de los que la mujer resulta víctima se encuentran contemplados tanto en la ley 26.485; como en el Código Penal.Es decir, si el hecho del que la mujer resulta víctima se encuentra únicamente descripto en el Código Penal -como sucede por ejemplo con las lesiones gravísimas-, entonces deberá instar la acción penal presentando su denuncia en el fuero penal. Lo mismo sucede en aquellos casos en los cuáles el hecho descripto por la mujer encuadra únicamente con lo previsto por la ley 26.485 -como es el caso de la violencia económica-, en este caso la judicialización será ante el fuero civil de la Ciudad Autónoma de Buenos Aires, o ante el fuero de familia en caso de que el hecho sucediera en la Provincia de Buenos Aires. Sin

\footnotetext{
${ }^{16}$ A los fines del desarrollo del presente trabajo, entenderemos como actores sociales tanto a las organizaciones no gubernamentales (ONGs), los movimientos sociales, los partidos políticos, los/as abogados/as particulares, entre otros; es decir, aquellos actores no estatales.

${ }^{17}$ La relevancia es tal que, por ejemplo, la Comisión sobre Temáticas de Género de la DGN ha reconocido que el número de casos nuevos patrocinados durante 2016 se incrementó en los momentos en que se dio mayor difusión a la problemática de la violencia de género, especialmente por la difusión masiva en los medios de comunicación así como por las convocatorias organizadas por el colectivo Ni Una Menos y el Paro Nacional de Mujeres (Comisión sobre Temáticas de Género, 2016, p. 4).
} 
González, A. D. y Varela, N. Violencia unificada y justicia fragmentada: Un análisis crítico de las trayectorias de las causas de violencia de género. Derecho y Ciencias Sociales. Mayo-Octubre 2019. № 21. Pgs 186-213. ISNN 1852-2971. Instituto de Cultura Jurídica y Maestría en Sociología Jurídica. FCJ y S. UNLP

embargo, en aquellos casos en los cuáles la mujer es víctima de situaciones que se encuentran previstas tanto en el Código Penal como en la ley 26.485 -esto es amenazas, violencia física sin que lleguen a ser lesiones graves, abuso sexual sin acceso carnal, entre otros- entonces será la víctima quién deba decidir ante qué fuero iniciar las actuaciones. Asimismo, los/as jueces/zas de la justicia civil pueden derivar la denuncia a sede penal si los hechos denunciados configuran un delito.

En cuanto a las medidas de protección que se hubieren solicitado, para controlar su efectividad, las fiscalías deben solicitar la información tanto al juzgado civil como al penal que intervengan en el caso, y deben consultar si se registran medidas respecto del imputado en el Sistema Federal de Comunicaciones Policiales (Ministerio Público Fiscal, 2016, p. 18). Sin embargo, en los procesos en que no se configure un delito estipulado en el Código Penal, las causas finalizarán con las medidas de protección solicitadas. Como la ley 26.485 habla de medidas preventivas y no de medidas cautelares, las mismas pueden adoptarse aunque no haya una causa de fondo. Si bien sus características son similares a las de una medida cautelar, ya que se exige peligro en la demora y riesgo para la víctima, actúan de manera diferente al no ser accesorias de una causa principal o de fondo. En este caso, quien controlará la efectividad de tal medida, será el/la juez/a civil, pero en caso de que se incumpla tal medida, la desobediencia tramitará bajo la órbita de un juez penal, ya que desobedecer la orden de un funcionario público encuadra en el tipo penal previsto en el artículo 239 del Código Penal.

Es de destacar que, de las estadísticas de la OVD, uno de los principales organismos que recibe consultas por hechos de violencia, se desprende que de los casos que han recibido durante el primer trimestre del año 2017, el $99 \%$ ha sido derivado a la justicia civil, mientras que de ese total, solo el $75 \%$ ha ido a la justicia penal (Oficina de Violencia Doméstica, 2017, p. 2). Por lo tanto, queda un $24 \%$ de los casos que finalizan en la justicia civil y que, por ende, no tienen sentencias de fondo.Nuevamente, solo en el caso de incumplimiento ante una medida se derivará a la justicia penal por la configuración del delito de desobediencia.

La situación se torna aún más compleja y confusa para la mujer cuando el hecho denunciado se fragmenta y tramita en ambos fueros a la vez.

\section{El problema de la fragmentación de fueros}

Diversos son los problemas que acarrea que un mismo hecho se fragmente y tramite tanto en el fuero civil como en el fuero penal simultáneamente. 
González, A. D. y Varela, N. Violencia unificada y justicia fragmentada: Un análisis crítico de las trayectorias de las causas de violencia de género. Derecho y Ciencias Sociales. Mayo-Octubre 2019. № 21. Pgs 186-213. ISNN 1852-2971. Instituto de Cultura Jurídica y Maestría en Sociología Jurídica. FCJ y S. UNLP

En primer lugar, los objetivos que persiguen cada uno de ellos se encuentran en las antípodas. En el fuero penal, el Estado se apropia del conflicto de la víctima que dio origen al proceso con el fin de determinar si la persona imputada cometió o no el delito que se le reprocha y, en caso afirmativo, determina la pena a imponer. En cambio, en el fuero civil, el principio rector es la reparación.

Si bien muchas veces hay un mismo hecho que tramita en ambos fueros, se hace con una finalidad diferente: por ejemplo, en el caso de un accidente de tránsito, en el fuero penal se busca determinar la responsabilidad penal del conductor, mientras que ante el fuero civil el objetivo es obtener una reparación económica. Sin embargo, en el tema que nos ocupa hay un único objetivo: poner fin a la violencia de género. De esta manera, en determinados casos de violencia se solicitan medidas protectorias en el fuero civil mientras se tramita el proceso penal por la comisión de un delito. Aunque en general ante la solicitud de medidas protectorias interviene un juzgado civil, no hay nada que impida que el juez penal dicte tales medidas, ya que según la ley 26.485, cualquier juez/a, aún siendo incompetente, tienen la facultad de sancionar medidas preventivas ${ }^{18}$ con carácter urgente (cfr. $2^{\circ}$ párr., art. 22). Así, muchas veces, producto de la innecesaria tramitación de estas actuaciones de manera desarticulada, se generan respuestas contradictorias para la persona que denuncia. Asimismo, la intervención de diferentes organismos afecta a las denunciantes y víctimas de los hechos de violencia, que se ven obligadas a asistir a múltiples dependencias y a relatar reiteradamente el hecho en cuestión (Rossi, 2017, p. 1). Es decir, se replican los testimonios y los trámites que deben realizarse al respecto, pese a que el hecho en cuestión sea uno solo. Esto produce una revictimización de la mujer que denuncia, por los daños psicológicos o patrimoniales que sufre la víctima como consecuencia de la falta de una adecuada asistencia e información por parte del Estado. Además comprende los efectos traumatizantes derivados de los interrogatorios policiales o judiciales, la exploración médico-forense o el contacto con el ofensor en el juicio oral. En este sentido, es necesario decir que, en el ámbito judicial, la respuesta nunca puede ser un nuevo agravio para la mujer como lo es la victimización secundaria, ya que debe ser la justicia quien de resguardo a la víctima y no quien incremente las consecuencias perjudiciales que le causó el delito.

Esta fragmentación de las causas de violencia de género que puede ocasionar que una mujer por un mismo hecho deba concurrir tanto a la justicia penal (ya sea Contravencional de la CABA o Criminal Nacional o ambas a la vez) como a la justicia civil para obtener una respuesta a un

\footnotetext{
${ }^{18}$ Prohibición de acercamiento, exclusión de la residencia común, suspensión provisoria del régimen de visitas, entre otras.
} 
González, A. D. y Varela, N. Violencia unificada y justicia fragmentada: Un análisis crítico de las trayectorias de las causas de violencia de género. Derecho y Ciencias Sociales. Mayo-Octubre 2019. № 21. Pgs 186-213. ISNN 1852-2971. Instituto de Cultura Jurídica y Maestría en Sociología Jurídica. FCJ y S. UNLP

hecho de tal sensibilidad, fue tratado por la CSJN, quien siguiendo el criterio de la Procuración General de la Nación resolvió que los hechos deben investigarse conjuntamente ${ }^{19}$. En este sentido, resulta interesante analizar la resolución 1232/17 de la Procuración General de la Nación (en adelante PGN), a través de la cual se efectúan una serie de recomendaciones destinadas a que los/as operadores/as judiciales brinden una mejor respuesta a la problemática. Asimismo, se plantean como objetivos "asegurar que la actuación del MPF en casos de violencia doméstica contra las mujeres se ajuste al estándar de debida diligencia reforzada emergente de la Convención Interamericana para Prevenir, Sancionar y Erradicar la Violencia contra la Mujer (...) y de la jurisprudencia y recomendaciones de los organismos internacionales de derechos humanos"; y "prevenir la revictimización de las mujeres que hayan sufrido violencia doméstica y garantizar el pleno goce de sus derechos reconocidos por la ley $\mathrm{n}^{\circ} 26.485$, el Código Procesal Penal de la Nación y los instrumentos internacionales de derechos humanos". El primer capítulo, destinado al logro de dichos objetivos, trata sobre "los recaudos que deben adoptar las fiscalías para el registro y unificación de causas, con la finalidad de reducir el riesgo de revictimización para las mujeres damnificadas y posibilitar un abordaje integral de los hechos" (p. 3). Allí, la PGN advierte que

"la dispersión de causas multiplica los esfuerzos para las víctimas, que deben declarar varias veces sobre los mismos hechos y peregrinar por distintos despachos judiciales. Esto incrementa los riesgos de revictimización, la desconfianza de las víctimas en el sistema de justicia y la tendencia a abandonar el proceso. También impide analizar los hechos integralmente y valorar su gravedad, a la vez que obliga a duplicar los esfuerzos para investigar cuestiones íntimamente vinculadas, generando una inadecuada utilización de los recursos. Por estas razones, las fiscalías deben adoptar medidas especiales para registrar y unificar las causas por violencia doméstica contra las mujeres" (p. 13).

Por otro lado, la CSJN se expidió sobre la cuestión en el fallo G., C. L. s/ Lesiones agravadas y amenazas -incidente $\mathrm{n}^{\mathrm{o}} 1$ - (2016), en el que comparten los fundamentos vertidos en el dictamen de la Procuradora General de la Nación. En el caso se trataba de la denuncia de una mujer contra su ex pareja que tramitaba en el Departamento Judicial de Morón, mientras que en un Juzgado Correccional de Capital tramitaba otra denuncia de la víctima. En su dictamen, la Procuradora señaló que los actos de violencia deben ser investigados y juzgados de manera conjunta a fin de cumplir tanto los deberes del Estado como de procurar un adecuado acceso a la justicia para las

\footnotetext{
19 Al respecto, véanse fallos “Cazón, A. C. s/Art. 149 bis" (2013), "M., Claudio s/Inf. Art. 149 bis" (2013); "P., Mariana s/amenazas con armas anónimas” (2014).
} 
González, A. D. y Varela, N. Violencia unificada y justicia fragmentada: Un análisis crítico de las trayectorias de las causas de violencia de género. Derecho y Ciencias Sociales. Mayo-Octubre 2019. № 21. Pgs 186-213. ISNN 1852-2971. Instituto de Cultura Jurídica y Maestría en Sociología Jurídica. FCJ y S. UNLP

víctimas. Asimismo, sostuvo que las agresiones reiteradas que había sufrido la víctima, constituían un mismo conflicto de violencia de género, por lo cual ese conjunto de actos debían investigarse en forma conjunta, pese a haberse producido en distintas jurisdicciones, ya que la fragmentación de los hechos obstaculizaría la eficacia de la actuación de la justicia y revictimizaría a la damnificada, por someterla a declarar en distintas oportunidades y distintos tribunales sobre hechos que forman parte de un mismo conflicto.

Pese a estas recomendaciones, la fragmentación de las causas de violencia de género continúa, por lo que sus efectos perjudiciales para las mujeres víctimas continúan replicándose.

\section{El problema de la falta de especialización}

La normativa internacional a la que debe dar cumplimiento nuestro país, obliga a la existencia de una justicia especializada, la cual debe actuar con máxima diligencia tanto en la investigación como en el juzgamiento de los casos de violencia, aun cuando se trate de delitos leves ya que, en muchos casos, estos constituyen el inicio de un "espiral de violencia" (Rossi, 2017, p. 1). De esta manera, otro punto a tener en cuenta es la falta de capacitación de operadores/as judiciales en temáticas de género, ya que la efectividad del acceso a la justicia se relaciona no solo con el reconocimiento de derechos, sino también con la forma en que se desarrolla en los tribunales dicho derecho, cuyo contexto se encuentra marcado por las prácticas de los/as operadores/as jurídicos, las cuales traslucen la existencia o no de formación especializada en género por parte de estos/as (Rodríguez Luna y Naredo Molero, 2013, p. 171).

A partir de la encuesta "percepciones de operadoras y operadores acerca de la desigualdad de género en la justicia de la Ciudad Autónoma de Buenos Aires" realizada por el Observatorio de Género en la Justicia de la CABA, es posible analizar, de manera superficial, la igualdad de género al interior del Poder Judicial, en cuanto a la ocupación de los cargos de magistrados y magistradas: el $52,3 \%$ son varones y el $47,7 \%$ mujeres. Las mujeres constituyen el $46,1 \%$ del total de jueces y juezas, el 40,6\% de los/as fiscales, el 60,5\% de los/as defensores/as y el 50\% de los/as asesores/as tutelares. Al igual que sucede en el resto de las jurisdicciones de la justicia, la mayor proporción de mujeres magistradas se agrupa en los cargos de menor jerarquía. Aun así, las brechas son menos marcadas. En lo que hace a la percepción de los/as operadores/as judiciales acerca de si al interior del Poder Judicial se trata con igual respeto a ambos géneros, el $51 \%$ de las mujeres considera que sí, el $27,1 \%$ no está ni de acuerdo ni en desacuerdo con tal afirmación y el 21,9\% considera que no existe un trato igualitario; la percepción masculina 
González, A. D. y Varela, N. Violencia unificada y justicia fragmentada: Un análisis crítico de las trayectorias de las causas de violencia de género. Derecho y Ciencias Sociales. Mayo-Octubre 2019. № 21. Pgs 186-213. ISNN 1852-2971. Instituto de Cultura Jurídica y Maestría en Sociología Jurídica. FCJ y S. UNLP

respecto de que las personas operadoras del derecho son tratadas con el mismo respeto independientemente de su género es del 65,8\%. Estos datos nos permiten observar que, en menor medida, las desigualdades en razón de género se replican al interior del Poder Judicial.

A ello se suma que, según la encuesta, tan sólo la mitad de las personas encuestadas ha participado de algún tipo de capacitación en género, aproximadamente 4 de cada 10 varones y 5 de cada 10 mujeres. Sin embargo, dicha encuesta también arroja como resultado que la mitad de las personas encuestadas opina que en nuestra sociedad no existe igualdad entre varones mujeres. Al mismo tiempo, en igual proporción, las respuestas obtenidas revelan que las personas encuestadas creen que las magistradas y los magistrados están influenciadas/os por los estereotipos de género presentes en la cultura. También, de manera muy contundente, 9 de cada 10 manifiestan que creen que la magistratura tiene responsabilidad en la erradicación de las desigualdades basadas en el género (Kohen, Maffía y Ruiz, 2016, pp 157-160; 224-225). Es decir, menos de la mitad de los/as operadores/as jurídicos cuentan con una capacitación en temáticas de género, pese a que casi la totalidad de dicho universo es consciente de la responsabilidad que tiene en la erradicación de las desigualdades basadas en el género. Esta falta de capacitación repercute en el modo en que van a tratar a la mujer víctima de violencia de género, la cual, por el contexto particular en que se inserta la situación que padece, no debe ser asistida del mismo modo que las víctimas de otros delitos. Asimismo, "los juzgados y los equipos interdisciplinarios, parecen considerar quienes tienen experiencia concreta, deberían funcionar como una guardia médica, en la que gente muy experimentada diagnosticara de inmediato qué grado de urgencia reviste el caso aún antes de la intervención del juez" (ELA, 2009, p. 61).

En este sentido, la Relatora Especial sobre la violencia contra la mujer, recomendó a Argentina “(i)mpartir programas apropiados de concientización y capacitación sobre los derechos de la mujer a los agentes estatales, los miembros de los órganos legislativos, los jueces, los abogados, los agentes del orden, los trabajadores sociales y sanitarios, los docentes y la población en general" (A/HRC/35/30/Add.3), a su vez, en el mismo sentido se manifestó el Comité para la Eliminación de la Discriminación contra la Mujer (CEDAW/C/ARG/CO/7).

Fue recién a partir del femicidio que tuvo como víctima a Micaela García que se debatió en el Congreso sobre la necesidad de que todas las personas que integran los tres poderes del Estado reciban capacitación obligatoria en materia de género, lo que finalmente derivó en la sanción de la Ley 27.499. En los fundamentos de dicha ley se hace expresa mención a la necesidad de dar cumplimiento a la normativa internacional con jerarquía constitucional ya mencionada a lo largo 
González, A. D. y Varela, N. Violencia unificada y justicia fragmentada: Un análisis crítico de las trayectorias de las causas de violencia de género. Derecho y Ciencias Sociales. Mayo-Octubre 2019. № 21. Pgs 186-213. ISNN 1852-2971. Instituto de Cultura Jurídica y Maestría en Sociología Jurídica. FCJ y S. UNLP

de este trabajo, la Convención de Belém do Pará, así como también refieren a que "no basta sólo con transmitir el contenido normativo sino fundamentalmente proporcionar las herramientas que permitan visualizar las desigualdades estructurales de las mujeres, de modo de generar una práctica transformadora"

La necesidad de brindar capacitaciones en materia de género a operadores/as jurídicos/as es producto de que la misma no tiene lugar en la instancia formal de educación, es decir las facultades de derecho, a las que concurren los/as abogados/as que luego ocuparán cargos de poder o de toma de decisiones, como los/as magistrados/as, los/as legisladores/as, entre otros (Bergallo:2008) De alguna manera, esto se refleja en que durante años tanto la educación formal como la magistratura estuvieron ajenas a la problemática aquí analizada. Que los/as operadores/as jurídicos ejerzan la función para la que fueron designados con conocimientos y perspectiva de género, permitiría mejorar el servicio de justicia, ya que se evitarían muchas situaciones que son ocasionadas por la falta de conocimientos en la materia. Al respecto, resulta interesante analizar un caso relevado por un medio especializado que da cuenta de esta situación: Yanina Olmos denunció por primera vez a su ex pareja, Carlos Palavecino, en el año 2009 por violencia de género. En múltiples oportunidades lo había denunciado por haber sido golpeada, pero después, según narra, "no seguía las denuncias". Recién en el año 2016 la justicia le dicta a su ex pareja una restricción perimetral. Sin embargo, meses después, él intentó matarla al propinarle cinco puñaladas. Según informó la víctima a Cosecha Roja, antes que comenzara el juicio, el fiscal le propuso un acuerdo a Yanina: anular el juicio a cambio de darle la patria potestad de sus hijos. Si no aceptaba -le dijo- Palavecino iría a juicio acusado por el delito de lesiones. El Frente Popular Darío Santillán ${ }^{20}$, que acompañó a Yanina en el reclamo de justicia, denunció que el acuerdo era "parte de un entramado machista y patriarcal que (...) quiere dejar a las mujeres excluidas de la posibilidad de justicia, haciendo interpretaciones falaces de los hechos e intentando acallar la realidad". Yanina rechazó el acuerdo y el 4 de mayo de 2018, a diez años de que empezara la violencia, se dió inicio al juicio contra su ex pareja (Cosecha Roja (9 de mayo de 2018)

Este caso, al igual que muchos otros casos, pone de manifiesto una situación que atraviesan muchas mujeres que son víctimas de la violencia machista y que deciden denunciar a fin de que el Estado les brinde protección e investigue los hechos, las cuales acaban -erróneamenteasumiendo la culpa, como en el caso de Yanina, de considerar que el caso no fue resuelto

\footnotetext{
${ }^{20}$ Una organización social de base de Argentina.
} 
González, A. D. y Varela, N. Violencia unificada y justicia fragmentada: Un análisis crítico de las trayectorias de las causas de violencia de género. Derecho y Ciencias Sociales. Mayo-Octubre 2019. № 21. Pgs 186-213. ISNN 1852-2971. Instituto de Cultura Jurídica y Maestría en Sociología Jurídica. FCJ y S. UNLP

judicialmente o que la medida protectoria fue dictada recién a los siete años del primer hecho sufrido, porque ella "no siguió" el caso. La información obtenida nos permite afirmarqueuna capacitación en la materia le brindaría a los/as operadores/as jurídicos/as las herramientas para brindar una respuesta acorde a la gravedad de la situación. A esta altura, no podemos dejar de advertir que uno de los principios rectores del derecho penal es la expropiación a la víctima del conflicto por parte del Estado; por su parte el derecho civil se rige por los principios de contradicción, igualdad de partes e impulso procesal, esto último significa que recae sobre la parte demandante (en este caso la mujer víctima de violencia de género) la obligación de impulsar el proceso a fin de obtener un pronunciamiento. Dichos principios aplicados a los casos de violencia de género no hacen más que reforzar la situación de vulnerabilidad en la que se encuentra la mujer, al tiempo que atentan contra el derecho de tutela judicial efectiva.

Asimismo, nos interesa detenernos en un tema en particular, ya que consideramos que tal como está planteado hoy no es respetuoso de los derechos y dignidad de las víctimas de violencia de género, al tiempo que contribuye ampliamente a su re-victimización: La valoración de la prueba en el proceso penal. En este aspecto no se ha repensado el rol de la víctima de violencia de género, sino que se la trata como a cualquier otra víctima cuyo testimonio debe ser valorado como el de un testigo. Esta óptica no se condice con un enfoque de género, el cual propone una visión empoderadora de las mujeres que han vivido hechos de violencia (Rodríguez Luna y Naredo Molero, 2013, p. 180). En este sentido, los/as jueces/zas se apegan a conceptos puramente teóricos respecto del sistema de valoración de la prueba, poniendo en tela de juicio la credibilidad del testimonio de la víctima, cuando éste constituye el único objeto de prueba del proceso penal $^{21}$. Así, este sistema debe repensarse transversalmente de modo que, junto con las garantías del imputado, se respeten los derechos de las víctimas especialmente vulnerables, como lo son las mujeres víctimas de violencia de género.

De esta manera, se ha planteado la necesidad de implementar fueros especializados y unificados para dar respuestas adecuadas y coordinadas ${ }^{22}$, entendiendo no solo sobre la imposición de medidas de protección sino también sobre las causas de fondo, y que las mismas sean tratadas por operadores/as jurídicos/as formados/as con perspectiva de género. En concordancia, en su último informe en el 2017, la Relatora Especial sobre la violencia contra la mujer, recomendó

\footnotetext{
${ }^{21}$ Al respecto, véase Di Corleto, J. (2017).

22 En este sentido, el Ministerio de Justicia y Derechos Humanos de la Nación ha implementado un espacio denominado "Justicia 2020", en el marco del cual se propone como uno de los objetivos la unificación del fuero civil y penal para atender la problemática de violencia de género, mediante la implementación de un Modelo de Acción Integral.
} 
González, A. D. y Varela, N. Violencia unificada y justicia fragmentada: Un análisis crítico de las trayectorias de las causas de violencia de género. Derecho y Ciencias Sociales. Mayo-Octubre 2019. № 21. Pgs 186-213. ISNN 1852-2971. Instituto de Cultura Jurídica y Maestría en Sociología Jurídica. FCJ y S. UNLP

Argentina "(s)ubsanar la falta de un proceso sistemático y uniforme de incorporación y aplicación directa de la Convención sobre la Eliminación de Todas las Formas de Discriminación contra la Mujer y de la Convención de Belém do Pará a nivel federal y provincial" (A/HRC/35/30/Add.3).

Finalmente, se advierte la necesidad de (re)pensar el proceso tanto penal como civil con una perspectiva de género que, sin vulnerar las garantías del imputado o demandado, sea comprensivo de la situación de vulnerabilidad en la que se encuentran las mujeres que denuncian hechos de violencia de género.

\section{7 ¿Es la creación de un fuero unificado y especializado en violencia de género la solución?}

Rebobinando, una de las grandes problemáticas de nuestro país es la violencia de género. Frente a ello, como vimos, el Estado ha asumido obligaciones internacionales a través de las cuales se compromete a prevenir, sancionar y erradicar la violencia contra las mujeres; a nivel interno se han sancionado y promulgado diversas leyes tendientes a cumplir con tales objetivos. Asimismo, el compromiso de la sociedad civil se ha incrementado exponencialmente en los últimos años, lo que permitió visibilizar la problemática y crear índices y estadísticas que dan cuenta de la cantidad de casos de violencia de género que se dan año a año.

Además de la visibilización de esta problemática paralelamente hubo un crecimiento en la judicialización de los hechos de violencia de género, así como un incremento en la creación de nuevas leyes (por ejemplo, la reciente sanción de la Ley 27.501 que modifica la Ley de protección integral a las mujeres e incorpora al acoso callejero como una forma más de violencia contra la mujer) que ampliaron el campo de lo justiciable al entender como violencia de género a situaciones que hasta ese momento se encontraban naturalizadas en la sociedad, como por ejemplo la violencia psicológica o económica.

En este contexto, el rol del Poder Judicial es clave para entender cuál es la respuesta estatal frente a hechos ya ocurridos ${ }^{23}$. Como analizamos a lo largo del presente trabajo, algunos de los problemas que se observan en la judicialización de los casos de violencia de género son la simultánea o fragmentada intervención del fuero civil y penal, la revictimización de las mujeres y la falta de capacitación de los operadores judiciales en temáticas de género.

\footnotetext{
${ }^{23}$ En lo que hace a la prevención de la violencia de género habrá que analizar la implementación, o no, de políticas públicas, lo que en principio corresponde al Poder Ejecutivo. Por ejemplo, cfr. Mecanismo de Seguimiento de la Convención de Belém do Pará (2015).
} 
González, A. D. y Varela, N. Violencia unificada y justicia fragmentada: Un análisis crítico de las trayectorias de las causas de violencia de género. Derecho y Ciencias Sociales. Mayo-Octubre 2019. № 21. Pgs 186-213. ISNN 1852-2971. Instituto de Cultura Jurídica y Maestría en Sociología Jurídica. FCJ y S. UNLP

Otra situación que merece ser considerada es la que se origina cuando la mujer es víctima de múltiples hechos de violencia -como suele suceder ya que la violencia de género es ante todo una forma de dominación que se prolonga en el tiempo, pudiendo esta durar horas, días, meses, años- y decide denunciar cada uno de esos hechos, aún en el caso en que dichas denuncias no se fragmenten entre el fuero civil y el penal, dichas denuncias tramitarán conforme el juzgado que por turno corresponda, desvirtuando de esta manera la situación de violencia de género de la que es víctima la denunciante, ya que no se analizan todos los hechos como parte de dicha violencia, sino como hechos aislados.

Párrafo aparte merece la situación que tiene lugar cuando la justicia civil dicta una restricción perimetral, prohibición de acercamiento o cualquier medida protectoria tendiente a proteger la integridad psicofísica de la mujer, y esta es incumplida por el hombre sobre el que recae tal medida cautelar. Dicho incumplimiento, en abstracto, constituye el delito de desobediencia, contemplado en el artículo 239 del Código Penal, y es considerado un delito contra la Administración Pública, ya que el bien jurídico que busca proteger es el correcto desempeño de la función pública (comprensiva tanto de la actividad administrativa, judicial y legislativa), que es la libertad de acción de la autoridad pública. Se lesiona el orden de la Administración Pública atacando el libre ejercicio de la actividad funcional, cuando se resisten o desobedecen las órdenes impartidas por las autoridades, explica Andrés J. D’ Alessio (2004, p. 786). Sin embargo, cabe preguntarse si el imputado realmente buscó resquebrajar el buen funcionamiento de la Administración Pública o dicho incumplimiento se enmarca en el mismo ciclo de violencia de género que dio origen a la medida cautelar que incumplió y originó esta nueva causa por el delito de desobediencia. Es preciso tener en cuenta que en estos casos, tramitarán simultáneamente dos causas ante dos fueros distintos, en una de ellas la afectada será la mujer y en la otra la Administración Pública, pero el origen del conflicto será el mismo: la violencia de género. En este sentido, consideramos que la creación de un fuero único especializado en violencia de género permitiría un abordaje integral de la problemática, que analizaría la desobediencia y la violencia de género como dos caras de una misma moneda y no como hechos aislados.

Frente a ello surge la pregunta que da origen al presente apartado respecto de si la creación de un fuero especializado en violencia de género es la solución a los conflictos planteados. Al respecto, consideramos que la creación de un fuero especializado y unificado en la materia evitaría la fragmentación o desdoblamiento de las causas de violencia de género, lo que evitaría la revictimización de las mujeres que decidan denunciar los hechos sufridos, en casos como el 
González, A. D. y Varela, N. Violencia unificada y justicia fragmentada: Un análisis crítico de las trayectorias de las causas de violencia de género. Derecho y Ciencias Sociales. Mayo-Octubre 2019. № 21. Pgs 186-213. ISNN 1852-2971. Instituto de Cultura Jurídica y Maestría en Sociología Jurídica. FCJ y S. UNLP

planteado en el párrafo anterior permitiría abordar la desobediencia de una medida protectoria como parte de la violencia de género y no como un mero ataque a la Administración Pública, no se fragmentaría el conflicto sino que se lo atendería de manera integral, y lo mismo sucedería con aquellas mujeres que denuncian múltiples hechos producto de una única relación violenta. Desde un punto de vista pragmático, es más factible que los/as operadores/as judiciales que conformen dicho fuero se capaciten en temáticas de género, ya que serán las cuestiones sobre las que trabajarán diariamente, a diferencia del resto de los/as operadores/as judiciales para quienes la violencia de género será una de las tantas problemáticas en las que intervengan. La creación de un fuero especializado en la materia posibilitaría la idea de sistematizar y unificar protocolos, criterios y medidas, y sobre todo que la respuesta que reciba la víctima sea producto de una evaluación integral del conflicto.

Una objeción que podría hacerse a la creación de un fuero único y especializado para la violencia de género es que se podría producir un aislamiento de la temática respecto del resto de las problemáticas. Sin embargo, consideramos que, teniendo en cuenta que la violencia de género es producto de una situación de desigualdad estructural histórica en la que se encuentra la mujer y que esto atraviesa distintos ámbitos, es necesario que la temática sea tratada de forma integral en un fuero específico para la materia, lo que no obstaculizaría a que haya un tratamiento armónico con el resto de las problemáticas, y además colaboraría a mejorar el planeamiento de la política criminal.

Por otro lado, debemos considerar el derecho a recibir una tutela judicial efectiva, consagrado en el art. 25 de la $\mathrm{CADH}$, que establece en términos generales la obligación del Estado de proveer a los/as ciudadanos/as sometidos/as a su jurisdicción una debida protección judicial cuando algunos de sus derechos haya sido violado, siempre que ese derecho sea reconocido por la Convención, la Constitución o las leyes internas del Estado. De acuerdo con el caso Velásquez Rodríguez (1988), la CIDH ha establecido que dicha protección procede "cualquiera sea el agente" que haya vulnerado un derecho, incluso cuando éste fuera un particular, ya que en ese caso el Estado incumplió su obligación de evitar tal vulneración, por lo que si posteriormente no brindara protección judicial, la estaría "auxiliando" en cierto modo.

Ahora bien, si afirmamos "que un determinado derecho de la persona está vigente en un ordenamiento jurídico dado implica, como mínimo, que dicho ordenamiento le reconoce ciertos alcances exigiendo su respeto y establece algún mecanismo tendiente a garantizar esa vigencia. Es precisamente en esa dimensión garantizadora donde se destaca el papel esencial de los órganos jurisdiccionales" (Ottaviano, 2013, pp. 538-539). De esta manera, el rol de los/as 
González, A. D. y Varela, N. Violencia unificada y justicia fragmentada: Un análisis crítico de las trayectorias de las causas de violencia de género. Derecho y Ciencias Sociales. Mayo-Octubre 2019. № 21. Pgs 186-213. ISNN 1852-2971. Instituto de Cultura Jurídica y Maestría en Sociología Jurídica. FCJ y S. UNLP

operadores/as judiciales sería "dar pronta seguridad jurídica, amparo frente al desamparo, tutela frente a la indefensión, abriendo las compuertas de la jurisdicción y garantizando las libertades fundamentales a todos los habitantes" (Grillo, 2004). El contenido de este derecho comprende, en primer lugar, el derecho de acceso a la jurisdicción, es decir a promover la función jurisdiccional. En este primer momento, los/as jueces/zas tienen el deber de posibilitar el acceso de las partes al juicio, sin restricciones irrazonables (Grillo, 2004). En este sentido, consideramos que sería una "restricción irrazonable", por ejemplo, la obligación que se le presenta a la mujer de asistir a distintas jurisdicciones y fueros -lo que genera que haya una revictimización para la misma- en su búsqueda de una solución a una única problemática de la cual es víctima: la situación de violencia de género en la que se encuentra inmersa.

Así, consideramos que la creación de un fuero único especializado en violencia de género es la vía idónea para la satisfacción del derecho que todos/as los/as ciudadanos/as -pero en este caso específico, las mujeres- tienen a recibir una tutela judicial efectiva.

\section{Conclusión}

Finalmente, como conclusión preliminar surge que las actuales respuestas estatales ante la problemática de la violencia de género resultan insuficientes, por lo que entendemos que es necesario repensar la manera en que se abordan en el ámbito judicial las causas de violencia de género. Así, advertimos que la articulación de medidas para prevenir y erradicar estos hechos deben ser implementadas en conjunto y de manera coordinada: las políticas públicas diseñadas por el Estado deben orientarse a hacer efectivos los derechos consagrados en nuestro marco normativo.

Uno de los desafíos que se presentan es la implementación de fueros unificados y especializados. Asimismo, entendemos que, por la complejidad de la problemática y para tratar el conflicto de una manera integral, es necesario que los juzgados que intervengan en la temática cuenten con un equipo interdisciplinario para evaluar las causas desde distintas ópticas, lo que les permitiría brindar una respuesta con un mayor grado de efectividad $\mathrm{y}$, de esta manera, tratar de neutralizar el espiral de violencia en el que se encuentran insertas las mujeres y evitar la prosecución de la victimización secundaria. En este sentido, resulta de especial relevancia la formación que reciban los/as profesionales que intervengan en estas causas, por lo cual se presenta como necesario que reciban capacitación para llevar a cabo sus tareas con perspectiva de género. 
González, A. D. y Varela, N. Violencia unificada y justicia fragmentada: Un análisis crítico de las trayectorias de las causas de violencia de género. Derecho y Ciencias Sociales. Mayo-Octubre 2019. № 21. Pgs 186-213. ISNN 1852-2971. Instituto de Cultura Jurídica y Maestría en Sociología Jurídica. FCJ y S. UNLP

Finalmente, otra cuestión que se presenta es la necesidad de repensar desde un enfoque de género los principios que deben aplicarse a los procesos que tratan los hechos de violencia contra las mujeres, como la valoración de la prueba en los casos en que se configure un delito o la carga de impulsar procesalmente las causas que tramitan en el fuero civil, problemática que desaparecería con la implementación de un fuero único especializado en violencia de género.

\section{Bibliografía}

Amnistía Internacional (2007). Más riesgos y menos protección. Mujeres inmigrantes en España frente a la violencia de género. Madrid: Sección española de Amnistía Internacional.

Beck, I. y Romeo, M. (2016), $1^{o}$ indice nacional de Violencia Machista, Ciudad Autónoma de Buenos Aires: Campaña Contá la violencia machista.

Bergallo, P. (2008). "El género ausente y la enseñanza del derecho en Buenos Aires". En Rodríguez, M. y Asensio, R. (coords.). Una agenda para la equidad de género en el sistema de justicia, pp. 1-28, Ciudad Autónoma de Buenos Aires: Del Puerto/CIEPP.

Bergallo, P. y Moreno, A. (2017). "Primera parte. Nuevas institucionalidades de género en el sistema de justicia". En Bergallo, P. y Moreno, A. (coords.). Hacia políticas judiciales de género,pp. 23-236, Ciudad Autónoma de Buenos Aires: Jusbaires.

Bodelón, E. (1995). "Pluralismo, derechos y desigualdades: una reflexión sobre el género". En Derechos y libertades: Revista del Instituto Bartolomé de las Casas, 1995, Año 2, No. 5, pp. 201-213.

Consejo Nacional de Mujeres (2016). Plan Nacional de Acción para la Prevención, Asistencia y Erradicación de la Violencia contra las Mujeres 2017-2019, Ciudad Autónoma de Buenos Aires: CNM.

Comisión sobre temáticas de género (2016). Informe 2016. Ciudad Autónoma de Buenos Aires: Defensoría General de la Nación. Sitio web: http://www.mpd.gov.ar/pdf/Informe\%202016.pdf[Consulta 12/08/2017].

Corte Suprema de Justicia de la Nación: https://www.csjn.gov.ar/omrecopilacion/omfemicidio/homefemicidio.html [Consultado: 15/05/2019]

Costa, M. (2017). "Feminismos jurídicos en la Argentina”. Bergallo/Moreno (coords.), Hacia políticas judiciales de género, Ciudad Autónoma de Buenos Aires: Jusbaires. 
González, A. D. y Varela, N. Violencia unificada y justicia fragmentada: Un análisis crítico de las trayectorias de las causas de violencia de género. Derecho y Ciencias Sociales. Mayo-Octubre 2019. № 21. Pgs 186-213. ISNN 1852-2971. Instituto de Cultura Jurídica y Maestría en Sociología Jurídica. FCJ y S. UNLP

D' Alessio, A. J. (Dir.) (2004). Código Penal: Comentado y anotado: Parte especial (Arts. 79 a 306). Buenos Aires: La Ley.

Di Corleto, J. (2017). "Igualdad y diferencia en la valoración de la prueba: estándares probatorios en casos de violencia de género”. Di Corleto, J. (comp.). En Género y justicia penal, pp. 285-308, Ciudad Autónoma de Buenos Aires: Didot.

ELA - Equipo Latinoamericano de Justicia y Género (2009). Violencia familiar: Aportes para la discusión de politicas públicas y acceso a la justicia. Buenos Aires: ELA. Sitio web: http://www.ela.org.ar/a2/index.cfm?aplicacion=app $187 \& \mathrm{cnl}=14 \&$ opc $=49$ [Consulta: 12/08/2017].

ELA - Equipo Latinoamericano de Justicia y Género (2012). Más allá de la denuncia: Los desafios para el acceso a la justicia. Investigaciones sobre violencia contra las mujeres.http://www.ela.org.ar/a2/index.cfm?aplicacion=app187\&cnl=14\&opc=49 [Consulta: $12 / 08 / 2017]$.

Facio, A. y Fries, L. (2005). "Feminismo, género y patriarcado", en Academia, Revista de Enseñanza del Derecho, Año 3, No 6, pp. 259-294.

Facchi, A. (2005) "El pensamiento feminista sobre el Derecho: un recorrido desde Carol Gilligan a Tove Stang Dahl”, en Academia, Revista de Enseñanza del Derecho, Año 3, No 6, pp. $27-47$.

Federici, S. (2005). Calibán y la bruja: Mujeres, cuerpo y acumulación originaria. Buenos Aires: Tinta Limón.

Grillo, I. I. M. (2004). El derecho a la tutela judicial efectiva. Ciudad Autónoma de Buenos Aires: SAIJ. Sitio web: http://www.saij.gob.ar/iride-isabel-maria-grillo-derecho-tutela-judicialefectiva-dacf040088-2004/123456789-0abc-defg8800-40fcanirtcod [Consulta: 17/05/2018].

Heim, D. (2016). Mujeres y acceso a la justicia. Ciudad Autónoma de Buenos Aires: Didot.

INDEC (2018). Registro único de casos de violencia contra las mujeres (RUCVM): Resultados estadísticos 2013-2017. Ciudad Autónoma de Buenos Aires: INDEC. Sitio web: https://www.indec.gob.ar/uploads/informesdeprensa/rucvm_03 18.pdf[Consulta 16/05/2018].

Kohen, B., Maffía, D. y Ruiz, R. (coords.) (2016), El género en la justicia porteña: Percepciones sobre la desigualdad. Ciudad Autónoma de Buenos Aires: Jusbaires.

Ley 27.499 Fudamentos .Honorable Cámara de Diputados de la Nación. Página web: https://www.hcdn.gob.ar/proyectos/textoCompleto.jsp?exp=1776-D- 
González, A. D. y Varela, N. Violencia unificada y justicia fragmentada: Un análisis crítico de las trayectorias de las causas de violencia de género. Derecho y Ciencias Sociales. Mayo-Octubre 2019. № 21. Pgs 186-213. ISNN 1852-2971. Instituto de Cultura Jurídica y Maestría en Sociología Jurídica. FCJ y S. UNLP

2017\&tipo=LEY\&fbclid=IwAR0R4HF6q9eEaEkgPQXh0bPEg9rTogfqBHI9cGzfWP-revj-

x_IJz3b7dyw [Consultado: 15/05/2019]

Maciel, D. A. y Brito Prata, P. (2011). "Movilización por nuevos derechos y cambio legal: la Campaña por la Ley Maria da Penha”. En Política. Revista de Ciencia Política, 2011, Vol. 49, No. 1, pp. 139-170.

Mecanismo de seguimiento de la Convención de Belém do Pará (2015). Segundo Informe de Seguimiento a la Implementación de las Recomendaciones del Comité de Expertas del MESECVI. Washington DC: Comisión Interamericana de Mujeres.

Ministerio Público Fiscal (2016). Guía de actuación en casos de violencia doméstica contra las mujeres. Ciudad Autónoma de Buenos Aires: Ministerio Público Fiscal. Sitio web: https://www.mpf.gob.ar/direccion-general-de-politicas-de-genero/files/2016/11/Gu\%C3\%ADade-actuaci $\% \mathrm{C} 3 \% \mathrm{~B} 3 n-e n-c a s o s-d e-v i o l e n c i a-d o m \% \mathrm{C} 3 \% \mathrm{~A} 9$ stica-contra-las-mujeres.pdf

[Consulta 16/05/2018].

Ottaviano, S. (2013). "La tutela judicial en materia penal. Algunas cuestiones problemáticas". En Vigo, R. L. y Gattinoni de Mujía, M. (dir.). Tratado de derecho judicial, Tomo I, pp. 537584, Ciudad Autónoma de Buenos Aires: Abeledo Perrot.

Oficina de Violencia de Doméstica (2017). Estadísticas Primer Trimestre 2017. Ciudad Autónoma de Buenos Aires: OVD-CSJN.

Rodríguez Luna, R. y Naredo Molero, M. (2013). “El acceso a la justicia en los juzgados de violencia de género". En Bodelón, E. (comp.). Violencia de género y las respuestas de los sistemas penales, pp. 171-196. Ciudad Autónoma de Buenos Aires: Didot.

Rossi, F. (2017). "Violencia de género y acceso a la justicia: el fuero unificado y especializado como posible solución”. En Suplemento de Género, Diario DPI, 2017, Año 1, No. 1, pp. 1-3. Sitio web: http://dpicuantico.com/sitio/wp-content/uploads/2017/02/Doctrina-suple1.pdf[Consulta: $10 / 08 / 2017]$.

Sosa-Sánchez, I. A. (2017). "Fronteras múltiples: género, interseccionalidad y ciudadanía”. En Iberofórum: Revista de Ciencias Sociales de la Universidad Iberoamericana, 2017, Año XII, No. 23, pp. 84-101.

\section{$\underline{\text { Notas periodísticas }}$}


González, A. D. y Varela, N. Violencia unificada y justicia fragmentada: Un análisis crítico de las trayectorias de las causas de violencia de género. Derecho y Ciencias Sociales. Mayo-Octubre 2019. № 21. Pgs 186-213. ISNN 1852-2971. Instituto de Cultura Jurídica y Maestría en Sociología Jurídica. FCJ y S. UNLP

Agustina Paz Frontera (10 de mayo de 2019). Sobre el "crimen pasional" y el patriarcal: te maté porque era mía. Página web: http://latfem.org/sobre-el-crimen-pasional-y-el-patriarcal-temate-porque-era-

$\underline{\text { mia/?fbclid=IwAR0dWgboDshLH6ouRBrcaVlVmGYcGGDbBm2Qp9m3 ruYN9116yLinID21O }}$ k [Consulta: 12/05/2019].

Cosecha Roja (9 de mayo de 2018). Tres denuncias y un intento de femicidio. Sitio web: http://cosecharoja.org/diez-anos-de-violencia-machista-tres-denuncias-y-un-intento-defemicidio/ [Consulta: 18/05/2018].

Fernanda Jara (5 de diciembre de 2018). “¡Justicia por Lucía!”: cómo fue la marcha y el paro de mujeres en el centro porteño. Página web: https://www.infobae.com/sociedad/2018/12/05/justicia-por-lucia-como-fue-la-marcha-y-el-parode-mujeres-en-el-centro-porteno/ [Consulta: 12/05/2019].

Infobae (12 de diciembre de 2018). Tras la denuncia de Thelma Fardin, se multiplicaron las llamadas a la línea 144. Página web: https:/www.infobae.com/politica/2018/12/12/tras-ladenuncia-de-thelma-fardin-se-multiplicaron-las-llamadas-a-la-linea-

144https://www.pagina12.com.ar/162519-thelmas-miles [Consultado: 14/05/2019].

Luciana Sánchez (2018). Vamos todas a abortar. Página web: http://revistaanfibia.com/ensayo/vamos-todas-a-abortar/ [Consulta: 12/05/2019].

Martín Slipczuk (19 de diciembre de 2018). Es verdadero que las llamadas por violencia de género aumentaron tras la denuncia contra Juan Darthés. Página web: https://chequeado.com/verificacionfb/es-verdadero-que-las-llamadas-por-violencia-de-generoaumentaron-tras-la-denuncia-contra-juan-darthes/[Consultado: 14/05/2019].

Observatorio Ni Una Menos (26 de enero de 2018). 1 femicidio cada 29 hs. en el 2017. Página web http://www.observatorioniunamenos.org.ar/2018/01/26/1-femicidio-cada-29hs-en-el-2017/ [Consulta: 13/05/2018].

\section{$\underline{\text { Jurisprudencia }}$}

CIDH (2001), Informe No 54/01, Caso 12.051, María Da Penha Maia Fernandes vs. Brasil.

CIDH (2011), Informe $\mathrm{N}^{\mathrm{o}} 80 / 11$, Caso $\mathrm{N}^{\mathrm{o}}$ 12.626, Jessica Lenahan (Gonzales) y otros vs. Estados Unidos. 
González, A. D. y Varela, N. Violencia unificada y justicia fragmentada: Un análisis crítico de las trayectorias de las causas de violencia de género. Derecho y Ciencias Sociales. Mayo-Octubre 2019. № 21. Pgs 186-213. ISNN 1852-2971. Instituto de Cultura Jurídica y Maestría en Sociología Jurídica. FCJ y S. UNLP

Corte IDH (2006), Caso Penal Miguel Castro Castro vs. Perú - Fondo, reparaciones y costas.

Corte IDH (2009), González y otras vs. México (“Campo Algodonero”) - Excepción preliminar, fondo, reparaciones y costas.

Corte IDH (2014), Velasquez Paiz y otros vs. Guatemala - Excepciones Preliminares, Fondo, Reparaciones y Costas.

Corte IDH (2014), Véliz Franco vs. Guatemala- Excepciones Preliminares, Fondo, Reparaciones y Costas.

CSJN (2012), “Cazón, A. C./art. 149 bis”, Sentencia del 27/12/2012 - Dictamen de la Procuradora General de la Nación del 23/11/2012.

CSJN (2013), "Recurso de hecho - Góngora, Gabriel Arnaldo s/causa N 14.092”, Sentencia del $23 / 4 / 2013$.

CSJN (2016), “G., C. L. s/ Lesiones agravadas y amenazas -incidente nº-", Sentencia del $17 / 05 / 2016$.

PGN (2015), “P., Mariana s/amenazas con armas o anónimas”, Dictamen del Procurador ante la CSJN del 1/4/2015.

TOC N 9 (2012), "Fernández Corina c/ Weber Javier s/ tentativa de homicidio", Sentencia del $23 / 10 / 2012$. 